\title{
MAST: A Mass Spectrometer Telescope for Studies of the Isotopic Composition of Solar, Anomalous, and Galactic Cosmic Ray Nuclei
}

\author{
W. R. Cook, A. C. Cummings, J. R. Cummings, T. L. Garrard, B. Kecman, \\ R. A. Mewaldt, R. S. Selesnick, E. C. Stone, and T. T. von Rosenvinge
}

\begin{abstract}
The Mass Spectrometer Telescope (MAST) on SAMPEX is designed to provide high resolution measurements of the isotopic composition of energetic nuclei from $\mathrm{He}$ to $\mathrm{Ni}(Z=2$ to 28 ) over the energy range from $\sim 10$ to several hundred $\mathrm{MeV} /$ nuc. During large solar flares MAST will measure the isotopic abundances of solar energetic particles to determine directly the composition of the solar corona, while during solar quiet times MAST will study the isotopic composition of galactic cosmic rays. In addition, MAST will measure the isotopic composition of both interplanetary and trapped fluxes of anomalous cosmic rays, believed to be a sample of the nearby interstellar medium.
\end{abstract}

\section{INTRODUCTION}

$\mathbf{T}$ HE Solar, Anomalous, and Magnetospheric Particle Explorer (SAMPEX), NASA's first Small Explorer (SMEX) mission, was launched in July 1992, carrying four instruments to measure the elemental and isotopic composition of energetic particles from solar and galactic sources. Two of these, the Mass Spectrometer Telescope (MAST) and the Proton Electron Telescope (PET; see [1]), were originally part of the Comprehensive Particle Analysis System (COMPAS) selected for flight on the U.S. spacecraft of the International Solar Polar Mission (ISPM). Although that spacecraft was canceled in 1981, midway through the construction of the MAST/PET instruments, MAST and PET were included on SAMPEX, providing the opportunity for MAST to measure the isotopic composition of several diverse energetic particle populations.

MAST is composed of an array of silicon solid state detectors of graduated thicknesses, including four positionsensitive detectors that measure the trajectories of inciden nuclei. A mass resolution of $\leq 0.25$ amu should be achieved for energetic nuclei from $\mathrm{He}$ to $\mathrm{Ni}$. Fig. 1 illustrates the energy range over which isotope resolution of these nuclei is possible. In this paper we describe the operation of MAST and summarize the scientific objectives that it will address. Descriptions of the other SAMPEX instruments (LEICA, HILT, and PET) can be found in accompanying articles by

Manuscript received August 3, 1992; revised January 21, 1993. This work was supported by the National Aeronautics and Space Administration under Contract NAS5-30704 and Grant NAGW-1919.

W. R. Cook, A. C. Cummings, J. R. Cummings, T. L. Garrard, B. Kecman, R. A. Mewaldt, R. S. Selesnick, and E. C. Stone are with the California Institute of Technology, Downs Laboratory 220-47, Pasadena, CA 91125.

T. T. von Rosenvinge is with the NASA/Goddard Space Flight Center, Greenbelt, MD 20771

IEEE Log Number 9208072.

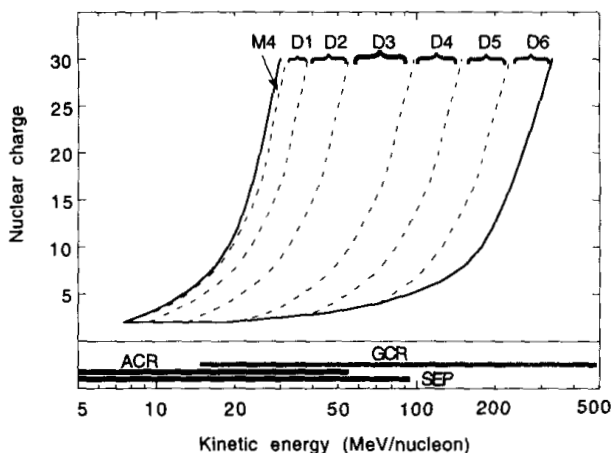

Fig. 1. The range of nuclear charge and energy/nucleon over which MAST is capable of resolving isotopes is shown between the solid curves. The dashed curves indicate the boundaries between energies where a nucleus stops in a given detector, for the case of normal incidence. The bars indicate the energy range for which solar energetic particles (SEP), anomalous cosmic rays (ACR), and galactic cosmic rays (GCR) typically show a significant flux.

Mason et al. [2], Klecker et al. [3], and Cook et al. [1]; an overview of the SAMPEX spacecraft and mission appears in Baker et al. [4].

\section{SCIENCE OBJECTIVES}

\section{A. The Isotopic Composition of Solar Energetic Particles}

Although the Sun contains $>99 \%$ of solar system material, we have only limited direct knowledge of its elemental composition and almost no direct knowledge of its isotopic composition. Spectroscopic observations of solar isotopes are difficult; there are isotope observations for only a few elements and the uncertainties are large. Almost all of the isotopic abundances in the Anders and Ebihara table of "solar system" abundances [5] are actually based on terrestrial material, while meteoritic measurements are the standard source of solar system elemental abundances.

Solar energetic particles (SEP) represent a sample of solar material that can be used to make direct measurements of the Sun's elemental and isotopic composition. Studies of the elemental composition of large solar flares (see, e.g., [6]) have shown that the relative abundances of elements in the solar corona with first ionization potential $\geq 10 \mathrm{eV}$ (including, e.g., $\mathrm{He}, \mathrm{N}, \mathrm{O}$, and $\mathrm{Ne}$ ) are depleted by a factor of $\sim 4$ compared to their abundances in the photosphere. Although the first 


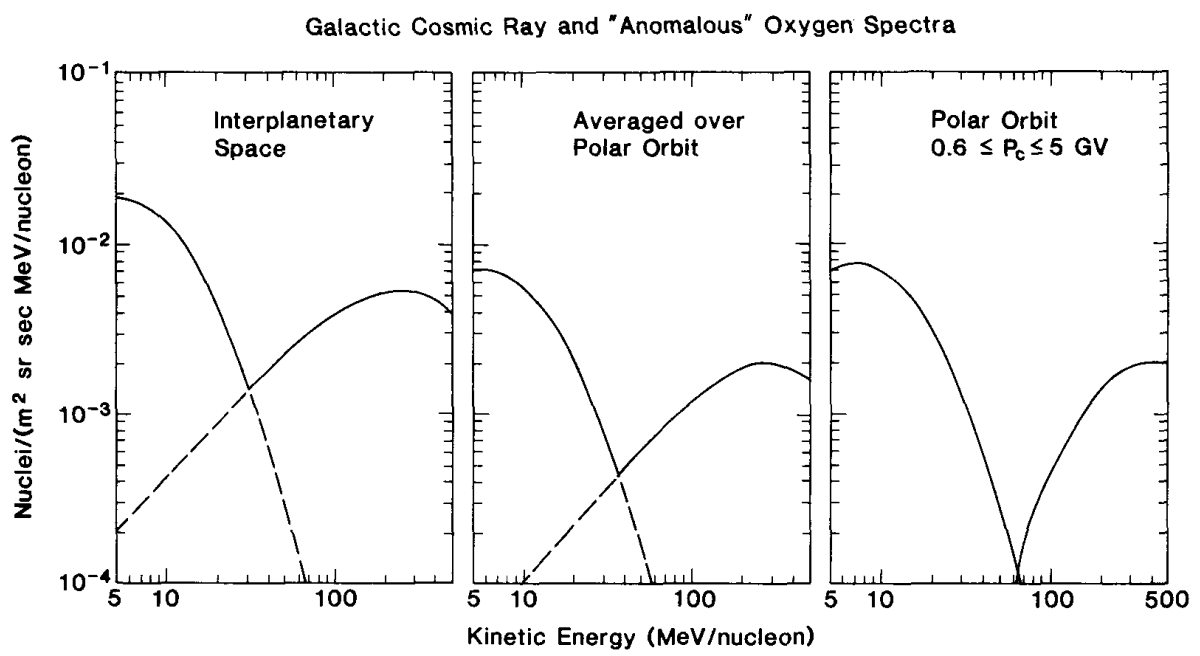

Fig. 2. The flux of oxygen in the anomalous cosmic rays (left, or lower energy, peak in each panel) and galactic cosmic rays at solar minimum. The three cases are for interplanetary space, a polar orbit average, and a polar orbit average limited to regions where the magnetic rigidity cutoff is $5 \mathrm{GV} \geq P_{c} \geq 0.6 \mathrm{GV}$. This figure illustrates how, by limiting the data set to regions of high magnetic rigidity, MAST can collect a sample of pure ACR, which are singly ionized.

measurements of the SEP isotopic composition were made in the late 1970's, such measurements are available for only a few elements in a limited number of solar events (see, e.g., [7] and references therein), and the uncertainties are large. SAMPEX will extend determinations of coronal composition to isotopes, as well as provide high precision measurements of elemental abundances over a broad range of energies. With its greatly improved collecting power over earlier instruments, MAST can substantially advance our knowledge of the Sun's isotopic composition.

\section{B. The Isotopic Composition of Anomalous Cosmic Rays and the Local Interstellar Medium}

Observations of quiet-time cosmic rays below $\sim 40 \mathrm{MeV} /$ nuc offer a unique opportunity to study matter from interstellar space. During solar minimum conditions there are several elements ( $\mathrm{He}, \mathrm{C}, \mathrm{N}, \mathrm{O}, \mathrm{Ne}, \mathrm{Ar}$, and perhaps $\mathrm{H}$ ) whose energy spectra have shown anomalous increases in flux above the quiet time galactic cosmic ray spectrum (see, e.g., [8], [9]). This so-called "anomalous cosmic ray" (ACR) component is now thought to represent a sample of neutral interstellar particles that have drifted into the heliosphere, become ionized by the solar wind or UV radiation and then are accelerated to energies up to tens of MeV/nuc [10]. The solar wind termination shock is the probable site of this acceleration [11]. An important prediction of this model is that anomalous cosmic rays should be singly ionized, in comparison to galactic cosmic rays which are essentially fully stripped. Strong evidence supporting this prediction was recently provided by Adams et al. [12], who reported a charge of +1 for ACR oxygen. On SAMPEX, HILT and MAST will measure more directly the charge states of anomalous $\mathrm{He}, \mathrm{N}, \mathrm{O}$, and $\mathrm{Ne}$ using the geomagnetic field as a rigidity filter (see discussion in [3]).

Because the ACR component apparently represents a direct sample of the local interstellar medium, it carries important information about galactic evolution in the solar neighborhood over the time interval since the formation of the solar nebula-information that can be obtained by comparing the isotopic composition of ACR nuclei with that of solar system nuclei, including those measured by MAST in large solar flares. Radio and optical spectroscopy studies of interstellar matter indicate that interstellar abundances of isotopes such as ${ }^{13} \mathrm{C},{ }^{15} \mathrm{~N},{ }^{17} \mathrm{O}$, and ${ }^{18} \mathrm{O}$ may differ by a factor of $\sim 2$ from solar system abundances [13], [14], consistent with the predictions of some galactic evolution models.

The polar orbit of SAMPEX offers a significant advantage for studying ACR isotopes because the Earth's magnetic field can be used as a filter to select a pure sample of ACR nuclei, uncontaminated by solar or galactic cosmic ray nuclei, which are highly ionized. Fig. 2 illustrates an example of this "double-spectrometer" approach. Note that over the geomagnetic cutoff range from $\sim 0.6$ to $\sim 5 \mathrm{GV}$ (singly ionized) ACR nuclei with $\sim 15-40 \mathrm{MeV} /$ nuc can penetrate the Earth's field, but solar and galactic cosmic rays with $<50$ $\mathrm{MeV} /$ nuc are completely excluded. Thus a pure sample of ACR oxygen and other ACR ions is obtained, a significant advantage over earlier ACR isotope studies [15], [16]. The isotopic composition of anomalous $\mathrm{Ne}$ will be of particular interest, because of the diverse range of ${ }^{22} \mathrm{Ne} /{ }^{20} \mathrm{Ne}$ ratios observed in various samples of solar system material, and the factor of at least four excess of ${ }^{22} \mathrm{Ne}$ observed in galactic cosmic rays. As solar minimum conditions approach, MAST should observe $\sim 200$ ACR Ne nuclei per year.

\section{Investigations of Trapped Anomalous Cosmic Rays}

Recently, Grigorov et al. [17] reported evidence for the existence of a radiation belt that is comprised of trapped ACRs. This trapping, which was predicted by Blake and Friesen [18], apparently occurs when a singly charged anomalous cosmic ray with a rigidity somewhat above the geomagnetic cutoff 
penetrates deeply into the magnetosphere and loses some or all of its remaining electrons in the upper atmosphere, such that its resulting rigidity is suddenly below the trapping limit. The existence of this radiation belt, which apparently waxes and wanes over the solar cycle, provides opportunities for a number of exciting new studies by SAMPEX.

Although the study by Grigorov et al. [17] identified only trapped anomalous oxygen; it is expected that anomalous ions such as $\mathrm{N}, \mathrm{Ne}, \mathrm{Ar}$, and possibly even $\mathrm{He}$ will also be trapped. The intensity of the trapped oxygen flux can be up to $\sim 10^{3}$ times that in interplanetary space. These studies will be significantly enhanced by results from SAMPEX, which will provide both temporal and spatial resolution so that the trapped intensities can be mapped for the first time. Measurements of the composition of the trapped ACR's in this new radiation belt will be of particular interest, including searches for rarer anomalous species such as $\mathrm{C}$ and $\mathrm{Kr}$. Measurements by MAST may provide a new means of studying the isotopic abundances of the neutral interstellar gas.

\section{The Isotopic Composition of Galactic Cosmic Rays}

Galactic cosmic rays represent an accessible sample of matter that originates outside the solar system. The isotopic composition of this sample of matter contains a record of the nuclear history of cosmic rays, including their synthesis in stars, and subsequent nuclear interactions with the interstellar gas. Cosmic ray isotope observations have already revolutionized the interpretation of both cosmic ray origin and transport in the Galaxy by demonstrating that the isotopic composition of cosmic ray source material differs from solar system material, implying differences in their nucleosynthetic history, and by revealing that the mean cosmic ray lifetime is $\sim 10$ million years (see, e.g., [19])-a factor of $\sim 3$ longer than expected. In addition to earlier reported enhancements in the abundance of the neutron-rich isotopes of $\mathrm{Ne}, \mathrm{Mg}$, and possibly $\mathrm{Si}$ in cosmic ray source material (see, e.g., [20]), there are also recently reported excesses in the abundance of GCR ${ }^{18} \mathrm{O}$ [21] and ${ }^{60} \mathrm{Ni}$ [22]. MAST will extend isotope studies to additional elements with a collecting power several times greater than those of earlier satellite instruments.

\section{INSTRUMENT DESCRIPTION}

\section{A. Approach}

Resolution of isotopes in MAST is accomplished by a refinement of the standard technique [23] of measuring the energy loss $\Delta E$ of a particle penetrating a detector of thickness $L_{o}$ and its residual energy loss $E^{\prime}$ in another detector where the particle stops. To illustrate, the range of a particle of mass $M$, charge $Z$, and kinetic energy $E$ can be approximated by

$$
R=k\left(\frac{M}{Z^{2}}\right)\left(\frac{E}{M}\right)^{\alpha}
$$

Combining this with a similar equation for the residual range $R-L$ of the particle after traversing a pathlength $L=$

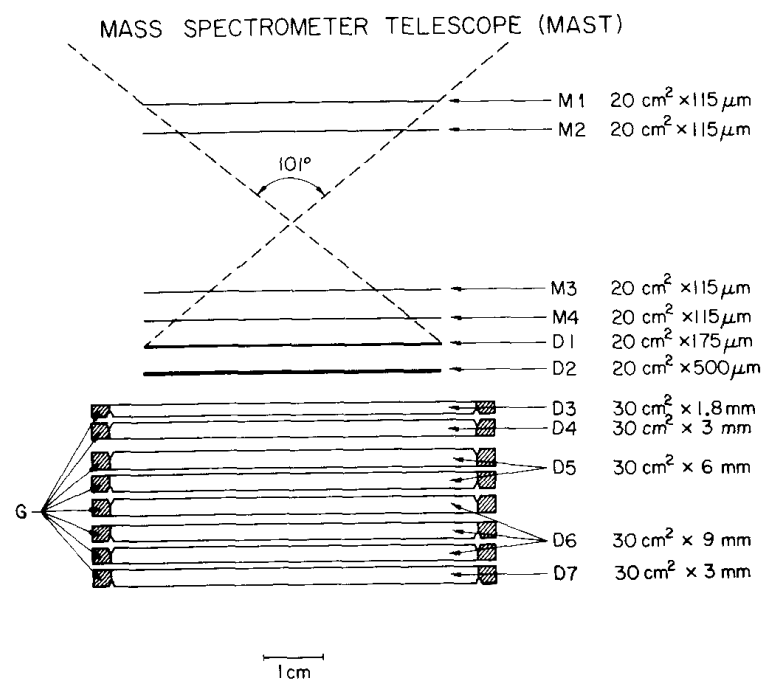

Fig. 3. A schematic representation of the detectors in MAST. The top four detectors (M1-M4) measure the position of the track of an incoming particle, and also its energy loss rate. The other detectors provide a precise measurement of the energy loss down to the detector in which the particle stops. The last detector in the stack (D7) and the guard rings (G) detect particles which exit the telescope. D5 and D6 are composite detectors of more than one wafer connected to a single amplifier. The dashed lines indicate the extreme trajectories intersecting the edge of D1, which defines the usable range for nuclei which stop in M4. See also Table I for more information.

$L_{o} \sec \Theta$, we find

$$
M=\left\{\frac{k\left[\left(\Delta E+E^{\prime}\right)^{\alpha}-\left(E^{\prime}\right)^{\alpha}\right]}{Z^{2} L_{o} \sec \Theta}\right\}^{1 /(\alpha-1)} .
$$

Isotope resolution, then, depends on precise measurements of the energy losses $\Delta E$ and $E^{\prime}$, the pathlength $L$ (which depends on $L_{o}$ and the zenith angle $\Theta$ ), and an accurate range-energy relationship.

\section{B. The Telescope}

The MAST detector stack, shown schematically in Fig. 3, consists of silicon detectors of graduated thickness. The upper section of the telescope is comprised of six surface-barrier devices, labeled M1 through D2, ranging in thickness from 115 $\mu \mathrm{m}$ to $500 \mu \mathrm{m}$. Four position-sensitive detectors (PSD's), M1 through $\mathrm{M} 4$, determine the trajectories of penetrating particles, as well as measuring their energy loss. The lower section has eight lithium-drifted (LiD) silicon wafers combined electronically into five detectors (D3 to D7). Table I summarizes the dimensions and other characteristics of the detectors.

Each of the LiD's has two circular grooves that divide the signal electrode into three annular regions, creating two independent concentric detectors and an outer dead area, a design used earlier in instruments on Voyager [24] and ISEE3 [23]. The outer active rings serve as anti-coincidence guards that permit discrimination against events in which a particle exits or enters through the side of the detector stack.

The central regions of all detectors except D7 are connected to charge-sensitive preamplifiers, shaping amplifiers, and 4095-channel ADC's. The center of D7 and the guard 
TABLE I

MAST DETECTORS

\begin{tabular}{|c|c|c|c|c|}
\hline $\begin{array}{l}\text { Detector } \\
\text { Name }\end{array}$ & $\begin{array}{l}\text { Type of } \\
\text { Measure- } \\
\text { ment }\end{array}$ & $\begin{array}{l}\text { Detector } \\
\text { Type }\end{array}$ & $\begin{array}{l}\text { Thickness } \\
(\mathrm{mm})\end{array}$ & $\begin{array}{l}\text { Diameter } \\
(\mathrm{cm})\end{array}$ \\
\hline M1 & $\begin{array}{l}\text { Trajectory, } \\
\Delta E\end{array}$ & PSD SB & 0.13 & 4.95 \\
\hline M2 & $\begin{array}{l}\text { Trajectory, } \\
\Delta E\end{array}$ & PSD SB & 0.12 & 4.95 \\
\hline M3 & $\begin{array}{l}\text { Trajectory, } \\
\Delta E\end{array}$ & PSD SB & 0.11 & 4.95 \\
\hline M4 & $\begin{array}{l}\text { Trajectory, } \\
\Delta E, E^{\prime}\end{array}$ & PSD SB & 0.11 & 4.95 \\
\hline D1 & $\Delta E, E^{\prime}$ & SB & 0.16 & 4.95 \\
\hline D2 & $\Delta E, E^{\prime}$ & SB & 0.5 & 4.95 \\
\hline D3 & $\Delta E, E^{\prime}$ & $\mathrm{LiD}$ & 1.8 & $\begin{array}{l}6.14 \\
\text { (center) }\end{array}$ \\
\hline D4 & $\Delta E, E^{\prime}$ & $\mathrm{LiD}$ & 3.1 & $\begin{array}{l}6.14 \\
\text { (center) }\end{array}$ \\
\hline D5 & $\Delta E, E^{\prime}$ & $\begin{array}{l}\mathrm{LiD}(2 \\
\text { wafers) }\end{array}$ & 6.2 & $\begin{array}{l}6.14 \\
\text { (center) }\end{array}$ \\
\hline D6 & $\Delta E, E^{\prime}$ & $\begin{array}{l}\text { LiD (3 } \\
\text { wafers) }\end{array}$ & 9.3 & $\begin{array}{l}6.14 \\
\text { (center) }\end{array}$ \\
\hline D7 & Penetration & $\mathrm{LiD}$ & 3.1 & $\begin{array}{l}6.14 \\
\text { (center) }\end{array}$ \\
\hline
\end{tabular}

PSD: Position Sensitive

SB: Surface Barrier

LiD: Lithium Drifted

regions of D3 to D7 are connected to preamplifiers, shaping amplifiers, and discriminators. The guards have a two-level discriminator set at $\sim 0.4$ and $5 \mathrm{MeV}$. D7 is used to identify those high-energy events in which a particle penetrates entirely through the detector stack.

The MAST telescope has two windows, spaced $1.5 \mathrm{~cm}$ apart, which provide electrical shielding and protection from micrometeorites and sunlight. The outer window is $0.025 \mathrm{~cm}$ thick, and the inner $0.076 \mathrm{~cm}$ thick. Both are made of Kapton, aluminized on the inner surface and coated with indiumtin oxide on the outer surface. A $0.125 \mathrm{~cm}$ thick aluminum collimator, with its rim $4.15 \mathrm{~cm}$ above M1, shields the front detectors from low energy particles incident at wide angles without blocking the $101^{\circ}$ viewing angle of the telescope.

\section{The Trajectory System}

The pathlength, $L$, through the $\Delta E$ detectors varies due to particles' varying angles of incidence and also due to nonuniformities in the detector thickness. Calibrations with $\mathrm{Si}$ and $\mathrm{Fe}$ beams at the Lawrence Berkeley Laboratory (LBL) Bevalac heavy ion accelerator have provided maps of these thickness variations. Thus, a trajectory measurement allows an accurate calculation of the pathlength through each of the $\Delta E$ detectors.

Detectors M1 to M4 are each a one-dimensional PSD. On one side of these devices the electrode consists of 93 strips, with $0.5 \mathrm{~mm}$ pitch, interconnected through $19 \mathrm{ohm}$ resistors to form a 92 resistor chain. A current pulse from the passage of a charged particle is divided into two charge-sensitive preamps on opposite ends of the divider network, with each of the pulse- heights (X1 and X2) approximately inversely proportional to the distance of the impacted strip from its end. The sum $X 1+X 2$ is then a measure of total energy loss in the detector, and the one-dimensional position coordinate, $X$, is given by the ratio $X=X 1 /(X 1+X 2)$. Because of the signal shaping time constants employed, there is a "ballistic deficit" in the summed charge (easily correctable) which varies with position, reaching a maximum of $\sim 10 \%$ for particles passing through the center strip of the detector. The strips on adjacent detectors are perpendicular to each other so that a trajectory can be calculated from the two X, Y coordinate pairs.

\section{Electronics}

MAST uses the signal processing technology originally developed for the Caltech EIS experiments (flown on IMPs 7 and 8 ) and further developed in thin-film hybrids for the Caltech HIST experiment on ISEE-3 [23]. The instrumentation consists of independent, parallel signal processing chains for each digitized parameter. Because these signal processors operate in a "normally open" linear gate mode, delay lines in the signal path, along with their attendant nonlinearity, poor temperature coefficient, and extra weight, are eliminated. The 4095-channel ADCs operate over a dynamic range of up to $1000:: 1$ (full scale :: threshold). Double integration, double differentiation, single pole RC shaping is used.

Each of the ADCs also has two digital discriminators whose outputs are used (in combination with those from the other triggered detectors) to classify events. The A discriminator is set for each detector at an energy just above the maximum possible energy deposit by a proton at maximum angle. The $\mathrm{B}$ discriminator is set just above the maximum energy deposit by an alpha particle at maximum angle. A He nucleus $(Z=2)$ will trigger at least one A discriminator if it stops in the telescope, and a $Z>2$ nucleus will trigger both $\mathrm{A}$ and $\mathrm{B}$ discriminators. The logical OR of all the A discriminators and the OR of all the $B$ discriminators sorts the events into one of three element categories: $Z=1, Z=2$, and $Z>2$. Events with $Z>2$ are given highest priority for readout, and of these, events with the deepest triggered detector have the highest priority. The next highest priority events, where D7 is triggered, are called penetrating events. Table II summarizes the logic requirements and event priorities.

MAST shares with PET [1] a common instrument housing and structural support. They also share a low voltage power supply assembly (LVPSA) which is contained in an external box. The power to each instrument is switched separately by relays in the LVPSA, controlled by the instrument Data Processing Unit (DPU, see [25]). Power to the LVPSA is controlled by the SAMPEX spacecraft.

\section{E. MAST Data}

The scientific data from MAST can be divided into two types, "event data" and "rate data." To generate an "event" in MAST a particle must satisfy the coincidence equation $\mathrm{M} 1 \cdot \mathrm{M} 2 \cdot \mathrm{M} 3 \cdot \mathrm{M} 4$. For such events we measure the trajectory in M1 through M4, and the individual energy losses in M1 through D2 and the centers of D3 through D6. Each event 
TABLE II

MAST COINCIDENCE LOGIC

\begin{tabular}{|c|c|c|}
\hline Event Type & Readout Priority & Logic Equation \\
\hline Calibration & 1 & Calibration flag \\
\hline$Z \geq 3, \mathrm{R} 4-6$ & 2 & $\begin{array}{l}\mathrm{M} \cdot \mathrm{Z} 3 \cdot(\mathrm{D} 4+ \\
\mathrm{D} 5+\mathrm{D} 6) \cdot \overline{\mathrm{D} 7} \cdot \overline{\mathrm{G}} \overline{2}\end{array}$ \\
\hline$Z \geq 3, \mathrm{R} 3$ & 3 & 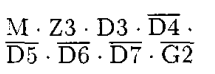 \\
\hline$Z \geq 3, \mathrm{R} 2$ & 4 & $\frac{\mathrm{M} \cdot \mathrm{Z3} \cdot \mathrm{D} 2 \cdot \overline{\mathrm{D} 3} \cdot \overline{\mathrm{D} 4}}{\overline{\mathrm{D}} 5} \cdot \overline{\mathrm{D} 7} \cdot \overline{\mathrm{G} 2}$ \\
\hline$Z \geq 3, \mathrm{R} 1$ & 5 & $\frac{\mathrm{M} \cdot \mathrm{Z3} \cdot \mathrm{D} 1 \cdot \overline{\mathrm{D} 2} \cdot \overline{\mathrm{D} 3} \cdot}{\overline{\mathrm{D}} 4 \cdot \overline{\mathrm{D} 5} \cdot \overline{\mathrm{D} 6} \cdot \overline{\mathrm{D} 7} \cdot \overline{\mathrm{G} 2}}$ \\
\hline$Z \geq 3, \mathrm{Ro}$ & 6 & $\overline{\mathrm{M}} \cdot \mathrm{Z3} \cdot \overline{\mathrm{D}} 4 \cdot \overline{\mathrm{D}} 5 \cdot \overline{\mathrm{D}} 6 \cdot \overline{\mathrm{D}} 2 \cdot \overline{\mathrm{D}} 3 \cdot$ \\
\hline Penetrating & 7 & $\mathrm{M} \cdot \mathrm{D} 7 \cdot \overline{\mathrm{G} 2}$ \\
\hline$Z=2$ & 8 & $\mathrm{M} \cdot \mathrm{Z} 2 \cdot \overline{\mathrm{D} 7} \cdot \overline{\mathrm{G} 1}$ \\
\hline$Z=1$ & 9 & $\mathrm{M} \cdot \mathrm{Z} 1 \cdot \overline{\mathrm{D} 7} \cdot \overline{\mathrm{G} 1}$ \\
\hline \multicolumn{3}{|c|}{$\begin{array}{l}\text { " }\{-\} " \text { means logical complement," }+" \text { means logical OR, and "." } \\
\text { means logical AND. }\end{array}$} \\
\hline \multicolumn{3}{|c|}{$\begin{array}{l}\text { "M" means that each of the matrix detectors is triggered, so that } \\
\text { a valid trajectory is obtained. }\end{array}$} \\
\hline \multicolumn{3}{|c|}{$\begin{array}{l}\text { "Z3" is the OR of the detector B discriminator levels, signifying } \\
Z>2 \text {. }\end{array}$} \\
\hline \multicolumn{3}{|c|}{$\begin{array}{l}\text { " } \mathrm{Z} 2 \text { " is the } \mathrm{OR} \text { of the detector A discriminator levels } \cdot \overline{\mathrm{Z} 3} \text {, } \\
\text { signifying } Z=2 \text {. }\end{array}$} \\
\hline \multicolumn{3}{|c|}{ "Z1" is $\overline{\mathrm{Z} 2} \cdot \overline{\mathrm{Z}} \overline{3}$, signifying $Z=1$} \\
\hline \multicolumn{3}{|c|}{$\begin{array}{l}\text { "G1" is the OR of the low level guard discriminators }(\sim 0.4 \\
\mathrm{MeV}) \text {. }\end{array}$} \\
\hline
\end{tabular}

therefore has a trajectory measurement and anywhere from 3 to 9 measurements of the energy loss rate due to ionization. Such redundant measurements permit the identification of the charge, mass, and energy of incident nuclei, as well as identification of events which undergo charge or mass changing nuclear interactions within the material of the telescope.

Events are read out of MAST by the SAMPEX DPU, which formats them for telemetry by the SAMPEX data system [4]. The DPU imposes a "throttle" on the rate of MAST events that are accepted for telemetry, typically allowing 10 or more MAST events per second, with highest priority given to $Z>2$ events, as shown in Table II. Each instrument has a quota of the spacecraft memory in order to ensure that no instrument will be shut out in a high particle flux situation.

Because the telemetry rate is insufficient to transmit every event in large solar flares, event accumulators are used to count events during six second intervals. The rate data consist of the trigger frequency for individual detectors and discriminators, and the counting rate of various detector combinations that satisfy the criteria for an event (e.g. the event types listed in Table II). For example, the counting rates of both $Z=2$ and $Z>2$ nuclei are monitored in seven separate energy intervals every 6 seconds. In addition, the counting rate of a continuous pulser is monitored to measure instrument "livetime." The individual detector counting rates are sampled over a 6-second period every 96 seconds. Such data are used to normalize particle fluxes and monitor instrument health. All of the coincidence equations and some of the discriminator levels can
TABLE III

MAST RESOURCES

\begin{tabular}{ll}
\hline Mass & $7.5 \mathrm{~kg}$ (including PET) \\
Power & $3.3 \mathrm{~W}$ \\
Bit Rate & $1430 \mathrm{bps}$ (orbit average) \\
\hline
\end{tabular}

be modified by command to allow for the possibility of noisy or failed detectors, and to optimize the instrument's response to $Z>2$ isotopes.

MAST has a built-in internal calibrator that can be initiated either periodically (every 6.8 hours) or by command (see also [1]). The calibrator includes an 8-bit DAC that provides a reference voltage to the internal test-pulsers for each of the signal channels. These are stimulated both individually and in combination in order to perform limited tests of the coincidence logic, measure the thresholds of the various discriminators, and check the gain, linearity, and long-term stability of the ADCs. Calibration "events" are flagged and stored in a special buffer for readout along with the regular data.

\section{F. Resources}

The MAST and PET instruments on SAMPEX are housed in a common box of dimensions $25 \mathrm{~cm} \times 36 \mathrm{~cm} \times 17 \mathrm{~cm}$, with power provided by a supply housed in a separate box of dimensions $13 \mathrm{~cm} \times 12 \mathrm{~cm} \times 6 \mathrm{~cm}$. Table III summarizes the spacecraft resources required by MAST. Although they share a common power supply, MAST and PET are electrically isolated. The peak power drawn by the instruments during large flares may increase by $\sim 25 \%$. MAST's average data rate of $1430 \mathrm{bits} / \mathrm{second}$ corresponds to $\sim 6$ transmitted events per second averaged over the orbit, but MAST will achieve significantly higher data rates during limited periods of activity through data management by the SAMPEX DPU. During launch operations both the MAST and PET telescopes were acoustically and thermally protected by a common 0.08 $\mathrm{cm}$-thick $\mathrm{Al}$ cover that was opened by command prior to instrument turn-on.

\section{G. Mass Resolution and Calibrations}

The design of MAST reflects the evaluation and optimization of all parameters affecting the mass resolution and collecting power, taking into account available resources. Statistical uncertainties due to fluctuations in the $\Delta E$ measurements are minimized by making anywhere from 3 to 9 measurements of $\Delta E$. The trajectory measurements provided by the PSDs, along with detector thickness maps obtained at the Bevalac, will allow determination of the pathlength $L$ to an accuracy of $\sim 0.1 \%$. Electronic contributions to the mass resolution are expected to be negligible. Detailed evaluations show that MAST should achieve an rms mass resolution of $\sigma_{m} \leq 0.25 \mathrm{amu}$ for nuclei from $\mathrm{He}$ to $\mathrm{Ni}(Z=2$ to 28$)$, sufficient, in principle, to separate adjacent isotopes that differ in abundance by a factor of 100 to 1 .

Because present knowledge of range energy relations for heavy ions is inadequate, MAST was exposed to beams of ${ }^{28} \mathrm{Si}$ and ${ }^{56} \mathrm{Fe}$ (and their fragments) at the LBL Bevalac to calibrate 


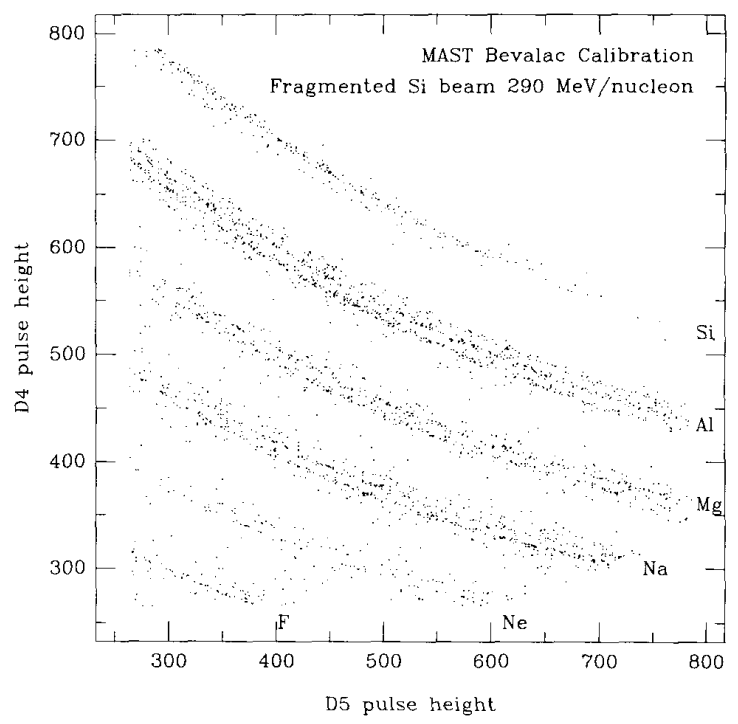

Fig. 4. Raw data from a calibration of MAST at the LBL Bevalac heavy ion accelerator. The plot shows the uncorrected pulse heights from D4 vs. D5, for a fragmented silicon beam at an incidence angle of $7.5^{\circ}$. Isotope tracks are clearly visible for elements from $F$ through Si. Corrections for thickness variations in the detectors obtained from this calibration will further improve the resolution of isotope tracks. The beam energy was about $290 \mathrm{MeV} /$ nucleon, but the target thickness was adjusted to produce particles stopping in D5.

directly the individual isotope tracks. Fig. 4 shows an example of such calibration data in a $\Delta E$ by $E^{\prime}$ plot of raw data from D4 and D5. The isotope resolution demonstrated here will be further improved when the measurements from earlier detectors (M1 to M4 and D1 to D3) are included in the mass determination, and when corrections for detector thickness variations are employed. Preliminary analysis of accelerator data shows a mass resolution of less than 0.2 amu for ${ }^{28} \mathrm{Si}$ nuclei.

The accelerator measurements will also permit mapping of the thickness of insensitive layers of material between the active detector volumes. Measurements with alpha particle sources show that "deadlayers" are of the order of $30 \mu \mathrm{m}$ for the LiD detectors (on the signal, or grooved, side), and less than $1 \mu \mathrm{m}$ for the surface barrier detectors. In addition, there is $13 \mu \mathrm{m}$ of aluminized mylar placed between facing signal sides of the LiD detectors in order to prevent crosstalk. Particles stopping in the deadlayers can be identified by their position on $\Delta E$ vs. $E^{\prime}$ plots such as Fig. 4.

\section{H. Collecting Power}

Fig. 5 shows the expected geometry factors of MAST for analysis of $\mathrm{He}, \mathrm{C}, \mathrm{Si}$, and $\mathrm{Fe}$ isotopes as functions of energy/nucleon, based on Monte Carlo simulations of the instrument's response. Because of the requirement to trigger the thresholds on the matrix detectors, MAST is less sensitive to low $\mathrm{Z}$ nuclei (lighter than about oxygen) than high $\mathrm{Z}$ nuclei.

Over the lifetime of SAMPEX, MAST is expected to collect significantly more anomalous cosmic ray events suitable for isotope analysis than all previous instruments combined, to

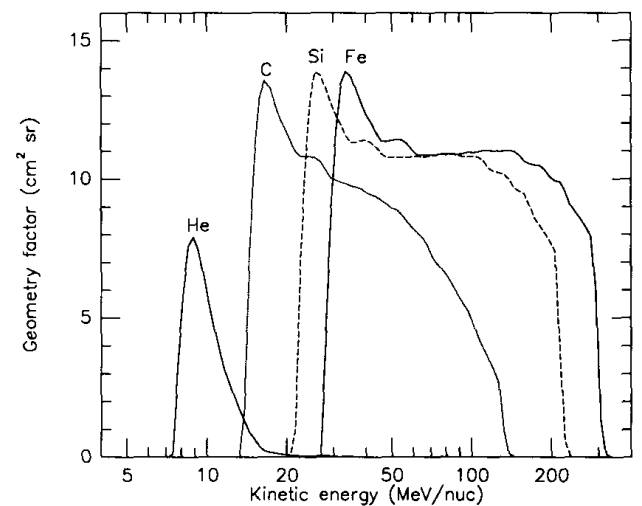

Fig. 5. The geometry factors of MAST for $\mathrm{He}, \mathrm{C}, \mathrm{Si}$, and $\mathrm{Fe}$ vs. energy/nucleon based on Monte Carlo simulations. These geometry factors are for nuclei which stop in the active detector volume and that produce sufficiently large signals in the PSDs to permit isotopic analysis (this requirement reduces the geometry factor for $\mathrm{He}$ and $\mathrm{C}$ at higher energies). Elemental analysis will have somewhat larger geometry factors than those shown here for the lighter elements, and will extend to somewhat higher energies for all elements. The shape of the curves is due to the varying size of the detectors and to the reduction of available incident angles as particles (with higher energy) stop deeper in the telescope.

make greatly improved measurements of the isotopic composition of solar energetic particles, and to obtain a large enough sample of galactic cosmic ray isotopes to permit a comparison of these three samples of galactic matter.

\section{ACKNOWLEDGMENT}

Significant contributions to the implementation of the MAST experiment were made by $\mathrm{H}$. Marshall and T. Harrington of MDH Ind.; D. Snyder and G. Takahashi of Ball Brothers Western Laboratories; D. Aalami of Space Instruments Inc.; N. Preketes of Perkin-Elmer Corporation, Applied Sciences Division; H. Crawford and the Bevalac staff at LBL; D. Mabry, S. Hansel, and K. Crawford of the Aerospace Corporation; W. Davis, G. Cooper, J. Kellogg, and H. Trexel of Goddard Space Flight Center; and W. Althouse, B. Gauld, K. Hargreaves, R. Vogt, and D. Williams of Caltech.

\section{REFERENCES}

[1] W. R. Cook, A. C. Cummings, J. R. Cummings, T. L. Garrard, B. Kecman, R. A. Mewaldt, R. S. Selesnick, E. C. Stone, D. N. Baker, T. T. von Rosenvinge, J. B. Blake, and L. B. Callis, "PET: A proton/electron telescope for studies of magnetospheric, solar, and galactic particles," IEEE Trans. Geosci. Remote Sensing, vol. 31, pp. 000-000, May 1993.

[2] G. M. Mason, D. C. Hamilton, P. H. Walpole, K. F. Heuerman, T. L. James, M. H. Lennard, and J. E. Mazur, "LEICA: A low energy ion composition spectrometer for the study of solar and magnetospheric heavy ions," IEEE Trans. Geosci. Remote Sensing, vol. 31, pp. 549-556, May 1993.

[3] B. Klecker, D. Hovestadt, M. Sholer, H. Arbinger, M. Ertl, H. Kastle, E. Kunneth, P. Laeverenz, and E. Seidenschwang, "HILT: A heavy ion large proportional counter telescope for solar and anomalous cosmic rays," IEEE Trans. Geosci. Remote Sensing, vol. 31, pp. 542-548, May 1993.

[4] D. N. Baker, G. M. Mason, O. Figueroa, G. Colon, J. G. Watzin, and R. M. Aleman, "An overview of the Solar, Anomalous, and Magnetospheric Particle Explorer (SAMPEX) Mission," IEEE Trans. Geosci. Remote Sensing, vol. 31, pp. 531-541, May 1993. 
[5] E. Anders and M. Ebihara, "Solar system abundances of the elements," Geochimica et Cosmochimica Acta, vol. 46, p. 2363, 1982.

[6] E. C. Stone, "Solar abundances as derived from solar energetic particles," AIP Conference Proceedings, 183, p. 72, 1989.

[7] R. A. Mewaldt and E. C. Stone, "Isotope abundances of solar coronal material derived from solar energetic particle measurements," Ap. J., 337 , p. $959,1989$.

[8] A. C. Cummings and E. C. Stone, "Elemental composition of the very local interstellar medium as deduced from observations of anomalous cosmic rays," Proceedings of the 21st International Cosmic Ray Conference (Adelaide), vol. 6, p. 202, 1990.

[9] E. R. Christian, A. C. Cummings, and E. C. Stone, "Evidence for anomalous cosmic ray hydrogen," Ap. J. 334, p. L77, 1988

[10] L. A. Fisk, B. Kozlovsky, and R. Ramaty, "An interpretation of the observed oxygen and nitrogen enhancements in low-energy cosmic rays," Ap. J. (Ltrs.), 190, p. L35, 1974.

[11] M. E. Pesses, J. R. Jokipii, and D. Ejchler, "Cosmic ray drift, shock wave acceleration, and the anomalous component of cosmic rays," $A p$. J. (Ltrs.), 246, p. L85, 1981.

[12] J. H. Adams, M. Garcia-Munoz, N. L. Grigotov M. A Kondratyeva, M. I. Panasyuk, Ch. A. Tretyakova, D. A. Zhuravlev, B. Klecker, G. M. Mason, R. E. McGuire, R. A. Mewaldt, and A. J. Tylka, "The charge state of the anomalous component of cosmic rays," Ap.J. (Ltrs.) 375, p. L45, 1991.

[13] A. A. Penzias, "Nuclear processing and isotopes in the galaxy," Science, vol. 208 , p. $663,1983$.

[14] P. G. Wannier, "Nuclear abundances and evolution of the interstellar medium," Ann. Rev. Astron. Astrophys., vol. 18, p. 399, 1980.

[15] R. A. Mewaldt, J. D. Spalding, and E. C. Stone, "The isotopic composition of the anomalous low-energy cosmic rays," Ap.J., 283, p. 450 1984.

[16] A. C. Cummings, E. C. Stone, and W. R. Webber, "Isotopic composition of anomalous cosmic ray neon," Proceedings of the 22nd International Cosmic Ray Conference, Dublin, Ireland, vol. 3, p. 362, 1991

[17] N. L. Grigorov, M. A. Kondratyeva, M. I. Panasyuk, Ch. A. Tretyakova, J. H. Adams, Jr, M Schulz, R. A Mewaldt, and A. J. Tylka, "Evidence for trapped anomalous cosmic ray oxygen in the inner magnetosphere," Geo. Res. (Ltrs.), vol. 18, p. 1959, 1991.

[18] J. B. Blake and L. M. Freisen, "A technique to determine the charge state of the anomalous low-energy cosmic rays," Proceedings of the 15th International Cosmic Ray Conference, Plovdiv, Bulgaria, vol. 2, p. 341 1977.

[19] J. A. Simpson and M. Garcia-Munoz, "Cosmic ray lifetime in the galaxy Experimental results and models," Sp. Sci. Rev., vol. 46, p. 205, 1988

[20] R. A. Mewaldt, "The abundances of isotopes in the cosmic radiation," AIP Conference Proceedings, vol. 183, p. 124, 1989.

[21] P. S. Gibner, R. A. Mewaldt, S. M. Schindler, E. C. Stone, and W. R. Webber, "The isotopic composition of cosmic ray $B, C, N$ and $O$ : Evidence for an overabundance of 18 O." Ap. J., 391, p. L89, 1992.

[22] R. A. Leske, B. Milliken, and M. E. Wiedenbeck, "The isotopic composition of iron-group cosmic rays," Ap. J. (Ltrs.), 390, p. L99, 1992.

[23] W. E. Althouse, A. C. Cummings, T. L. Garrard, R. A. Mewaldt, E. C. Stone, and R. E. Vogt, "A cosmic ray isotope spectrometer," Geoscience Electronics, vol. 16, pp. 204-207, 1978.

[24] E. C. Stone, R. E. Vogt, F. B. McDonald, B. J. Teegarden, J. H. Trainor, J. R. Jokipii, and W. R. Webber, "Cosmic ray investigation for the Voyager Missions; Energetic particle studies in the outer heliosphere-and beyond," Space Science Reviews, vol. 21, pp. 355-376, 1977

[25] D. J. Mabry, S. J. Hansel, and J. B. Blake, "The SAMPEX Dat Processing Unit (DPU)," IEEE Trans. Geosci. Remote Sensing, vol. 31 , pp. 572-574, May 1993.

Walter R. Cook received the B.S. degree in physics from Stevens Institute of Technology and the Ph.D. degree in physics from the California Institute of Technology.

He has been employed at Caltech's Space Radiation Laboratory since 1980 and is currently the principal electronics engineer and a Member of the Professional Staff. His research work has included studies of the composition of solar energetic particles on Voyager 1 and 2 and gamma-ray astronomy using the GRIP balloon-borne telescope, for which he serves as project scientist and electronics engineer. He was principal electronic engineer for the MAST/PET instruments on SAMPEX.

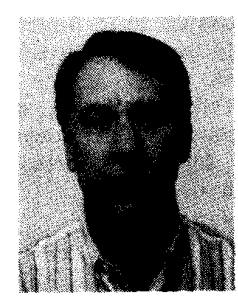

Alan C. Cummings received the B.A. degree in physics from Rice University in 1966. After a year at Churchill College, Cambridge University, he entered graduate school at Caltech where he received the Ph.D degree in physics in 1973.

$\mathrm{He}$ accepted the position of Research Scientist in Caltech's Space Radiation Laboratory in 1973 and now holds the position of Senior Scientist and Member of the Professional Staff. He has been responsible for hardware on the Voyager and ISEE3 missions and was Project Manager during the development of the Gamma Ray Imaging Payload balloon instrument, as well as serving as the MAST/PET Project Manager on the SAMPEX mission. His research interests include all aspects of the anomalous cosmic ray component. $\mathrm{He}$ is Co-Investigator on the Voyager and ACE missions.

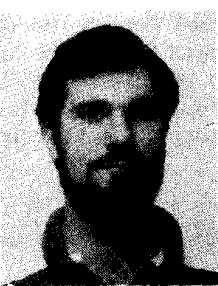

Jay R. Cummings received the Ph.D. degree from the University of Minnesota in 1989.

$\mathrm{He}$ is a Senior Research Fellow in Physics at the California Institute of Technology. His recent research includes investigation of the composition of particles trapped in the Earth's magnetosphere and of anomalous cosmic rays using the SAMPEX MAST and PET instruments, and studying fragmentation of relativistic beavy ions with the Ultra-Heavy Ion Cross-section collaboration.

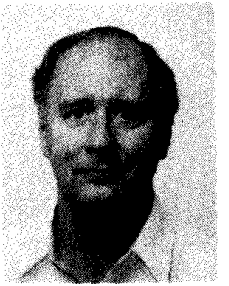

Thomas L. Garrard received the B.A. degree in physics from Rice University and the Ph.D. degree in physics from the California Institute of Technology.

He became a Staff Scientist at Caltech in 1972 and is now a Senior Scientist and Member of the Professional Staff. He is currently involved in SAMPEX, ACE, Galileo, Voyager, and other projects, with emphasis on composition of cosmic rays and solar energetic particles.

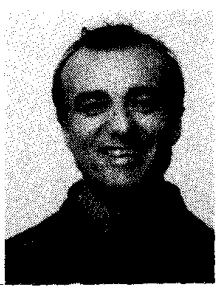

Branislav Kecman received the B.S. degree in electrical engineering from the University of Belgrade, Yugoslavia, in 1987, and the M.S.E.E. degree from the California Institute of Technology in 1989.

He worked as an Associate Electronics Engineer with the Space Radiation Laboratory at Caltech since 1989, where he is involved in design and testing of analog and digital electronic systems. For his efforts on the SAMPEX Spacecraft Integration and Test 'Team he was awarded NASA's Special Act Group Award in 1992.

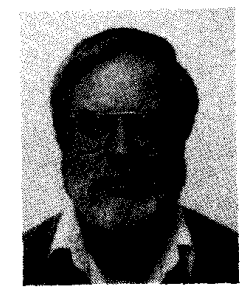

Richard A. Mewaldt received the Ph.D. degree in physics from Washington University, St. Louis, MO.

He has been a member of the Space Radiation Laboratory at the California Institute of Technology since 1971 . He is currently a Senior Research Associate in physics at Caltech. His research interests have centered on the study of energetic nuclei and electrons accelerated in solar flares, in interplanetary space, and in galactic cosmic rays, and on the development of high resolution spaceflight and balloon-borne instrumentation to measure the composition of these particles. $\mathrm{He}$ has been a Co-investigator on experiments for the NASA missions IMP-7 \& 8, ISEE-3 (ICE), ISPM, and ACE, and is a co-investigator on SAMPEX. 


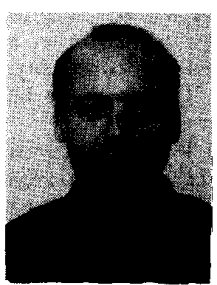

Richard S. Selesnick received the Ph.D. degree in space plasma physics from the Massachusetts Institute of Technology in 1988.

$\mathrm{He}$ is a Senior Research Fellow in Physics at the California Institute of Technology. His current research interests include the physics of planetary magnetospheres and cosmic rays.

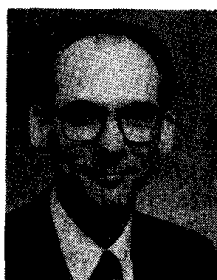

Edward C. Stone received the Ph.D. degree in physics from the University of Chicago in 1964.

He then joined the faculty of the California Institute of Technology, where he became Professor of Physics in 1976. He is currently the Director of the Jet Propulsion Laboratory and Vice President of Caltech. He has served as Project Scientist for the Voyager Mission to the outer planets since 1972. He has been a principal investigator on nine NASA spacecraft and a co-investigator on four others, including SAMPEX.
Tycho T. von Rosenvinge received the B.A. and Ph.D. degrees in physics from Amherst College and the University of Minnesota, respectively

In 1969 he came to NASA/Goddard Space Flight Center, where he is currently head of the Low Energy Cosmic Ray Group. He was Project Scientis for the International Sun-Earth Explorer ISEE-3 (1971-1983) and for the International Cometary Explorer, the first spacecraft to intercept a comet (1984-1989). He is currently Principal Investigator of the EPACT instruments on the GGS/WIND spacecraft. His scientific interests include the acceleration, composition, and transport of energetic particles in space, particularly particles from solar flares and the anomalous component. 